\title{
Evolutionary self-energy-loss effects in compact binary systems
}

\section{Importance of rapid rotation and of equation of state}

\begin{abstract}
N. K. Spyrou and N. Stergioulas
Astronomy Department, Aristoteleion University of Thessaloniki, 540.06 Thessaloniki, Macedonia, Greece

e-mail: spyrou@astro.auth.gr, niksterg@astro.auth.gr

Received 13 September 2000 / Accepted 9 November 2000

Abstract. The spin-down of a millisecond pulsar in a compact binary system leads to self-energy losses, that cause the binary's orbital period to increase (the effect being of first post-Newtonian (1PN) order). If the pulsar's period-derivative is not exceedingly small, this effect can become measurable in long-term high-precision timing measurements of the binary's orbital motion. We use rotating compressible spheroids to obtain an approximate, explicit expression for the orbital period derivative, due to spin-down, valid to 1PN order. This expression can be used to observationally constrain the pulsar's moment of inertia, and, combined with other observations, the high-density equation of state of compact stars. We apply our expression to representative models of millisecond pulsars in binary systems and demonstrate the importance of including higher-order rotational effects as well as the importance of the choice of equation of state, which both have been neglected, so far, in the literature. The computed increase in orbital period is larger, by as much as $32 \%$, when the higher-order rotational effects are included, while it can change by more than a factor of two between different plausible equations of state.
\end{abstract}

Key words. equation of state - stars: evolution - pulsars: general - rotation

\section{Introduction and motivation}

A large number of known millisecond pulsars are members of binary systems (see Phinney \& Kulkarni 1994; Lorimer 1998 for reviews). Nine new millisecond pulsars in binary systems have recently been discovered in the globular cluster 47 Tucanae (Camilo et al. 2000), and future observations are expected to yield an even larger number of such systems. Long-term timing observations of several relativistic binary pulsars have allowed the measurement of secular changes in the orbital period, caused by emission of gravitational radiation, with very high accuracy (see Kaspi 1999 for a recent review).

Various causes for secular changes in the orbital period of a binary pulsar have been examined to date. The first and traditional such study, in the case of a two-point-mass binary, has been based on the use of the quadrupole formula for the loss of orbital energy in the form of gravitational radiation and of the binary's Newtonian (Keplerian) orbital motion (Peters \& Mathews 1963). The above lowest-order result has been extended (Spyrou \& Papadopoulos 1985) to the general-relativistic 1PN approximation, with the aid of a similarly generalized quadrupole formula (Epstein \& Wagoner 1975) and of the binary's 1PN orbital motion (Spyrou 1981, 1983; see also
Blanchet \& Schäfer 1989). Of central importance to the above calculations is the fact that both members of the binary are treated as realistic bodies with finite dimensions, internal structure and internal motions, as opposed to the usual Newtonian point mass of the standard two-body Kepler problem. More specifically, the members are described in terms of the so-called inertial (or gravitational) mass, namely, their total mass-energy, and the corresponding position and velocity three-vectors. These parameters are valid to 1PN approximation and, initially, they were proposed by Spyrou (1977a,b, 1978; see also Caporali \& Spyrou 1981 for an inclusion of finite-size effects in a relativistic parametrized description of the realistic binary). As a consequence, the binary's total inertial mass manifests itself in the 1PN description of its orbital motion and, therefore, any of the members's characteristics of internal structure and motions can in principle affect its orbital motion and hence orbital period. Especially in the case of the binary pulsar B1913+16, it was proved (Spyrou \& Papadopoulos 1985) that, based on the then available observational data (Weisberg \& Taylor 1984), the relative 1PN correction to the orbital-period shortening, deduced from the above mentioned generalized quadrupole formula, was about five orders of magnitude smaller than the relative observational uncertainty. 
Another cause for secular changes in the orbital period of a binary or binary-participating compact star, are evolutionary changes in the compact star's total selfenergy (Spyrou 1985, 1987; see also Spyrou 1988, 1999 for the importance, as a cause of orbital-period changes, of the accretion-induced changes of binary-participating pulsar's period of axial rotation and radius). Especially in the case of the binary pulsar B1913+16, it was claimed (Spyrou 1987) that an accuracy improvement in the next decimal place of the then observed values (Weisberg \& Taylor 1984) of the orbital period's rate of change and of the associated uncertainty, would permit orbital-period shortening due to self-energy losses to be distinguished from that due to gravitational-radiation losses.

In this paper, we will focus on the secular spin-down of a pulsar (due e.g. to magnetic braking), that can cause a decrease in its rotational kinetic energy and thus, a corresponding change in the pulsar's gravitational mass. By conservation of the orbital angular momentum, this leads to an increase in the orbital period of the binary (Jeans 1924, 1928; Smarr \& Blandford 1976; Damour \& Taylor 1991). If the pulsar's period is only a few milliseconds and its spin-down rate is of the order of $10^{-19} \mathrm{~s} / \mathrm{s}$ (which is not uncommon for millisecond pulsars), then the induced secular increase in the orbital period may be measurable with current technology. Previous studies of this effect have used only lowest-order approximations for the selfenergy change of the pulsar, to obtain order-of-magnitude estimates of the effect (see e.g. Smarr \& Blandford 1976; Will 1981; Damour \& Taylor 1991). For example, the star was considered to be spherical, i.e. higher order rotational corrections to the shape of the star were neglected, and no particular equation of state was chosen. Here, we present a detailed description of mass-energy loss from a rapidly rotating pulsar, deriving an expression for the orbital period change that can be used to accurately constrain the pulsar's moment of inertia, from long-term timing measurements of the orbital period. Our treatment relies on the description of rapidly rotating neutron stars as compressible ellipsoids (Lai et al. 1993, hereafter LRS). We apply our results to typical millisecond pulsars in binary systems and derive constraints on their parameters, for the effect to be observable. Moreover, our results are derived consistently to first post-Newtonian order. The relation between mass-energy loss and orbital period increase, originally derived by Jeans (1924), assumed Newtonian gravity and isotropic mass-energy loss. The inclusion of 1PN terms in the description of the binary's orbit, changes the orbital period at $2 \mathrm{PN}$ order. Thus, Jean's relation is valid to $1 \mathrm{PN}$ order. Finally, we show that, including spin-orbit coupling in the description of the binary's orbit, changes the above relation by a term that can be neglected for typical binary systems.

The outline of the paper is as follows: In the next Sect. 2, we derive the self-energy loss of a rapidly rotating compact star, due to evolutionary spin-down, while in Sect. 3, we derive the corresponding orbital period change. In Sects. 4 and 5 we present lowest-order estimates and exact numerical results for the orbital period change and we conclude with a discussion of our results in Sect. 6 .

\section{Self-energy loss due to spin-down}

We will derive a relation between the spin-down rate of a pulsar and the resulting mass-energy loss, valid for rapidly rotating stars. The structure of rotating stars is approximated by compressible spheroids (introduced by LRS). These are approximate equilibrium configurations, the compressible generalization of Maclaurin spheroids (Chandrasekhar 1969), constructed using a variational method. The equation of state (EOS) has the usual zerotemperature polytropic form

$P=K \rho^{1+1 / n}$,

where $K$ is the polytropic constant and $n$ is the polytropic index. A polytropic index of $n=0.5$ corresponds to a relatively stiff EOS, while a polytropic index of $n=1$ corresponds to a relatively soft EOS. The rigidly-rotating, oblate spheroid has major axis $a_{1}$, minor axis $a_{3}<a_{1}$ and eccentricity

$e=\sqrt{1-\left(a_{3} / a_{1}\right)^{2}}$

If $\bar{m}$ is the baryonic mass of the spheroid and $R_{0}$ the radius of a nonrotating (spherical) star of the same baryonic mass, then the gravitational binding energy $W$ is

$W=-\frac{3}{5-n} \frac{G \bar{m}^{2}}{R_{0}} g(e)\left[g(e)\left(1-2 \frac{T}{|W|}\right)\right]^{n /(3-n)}$

where,

$g(e)=\left(1-e^{2}\right)^{1 / 6} \frac{\sin ^{-1} e}{e}$.

In (3), the ratio of rotational kinetic energy $T$ to $|W|$ depends only on the eccentricity $e$ and is given by

$\frac{T}{|W|}=\frac{3}{2 e^{2}}\left(1-\frac{e\left(1-e^{2}\right)^{1 / 2}}{\sin ^{-1} e}\right)-1$.

The internal energy $U$ of the spheroid is assumed to satisfy the virial relation

$\frac{3}{n} U+W+2 T=0$

and the total self-energy $\mathcal{E}=U+W+T$ is given by

$\mathcal{E}=\frac{3-n}{3} W\left(1-\frac{3-2 n}{3-n} \frac{T}{|W|}\right)$

The moment of inertia is

$I=\frac{2}{5} \kappa_{n} \bar{m} R_{0}^{2}\left(1-e^{2}\right)^{-1 / 3}\left[g(e)\left(1-2 \frac{T}{|W|}\right)\right]^{-\frac{2 n}{3-n}}$,

where $\kappa_{n}$ is a constant that depends on the equation of state (see LRS), with typical values $\kappa_{n}=(1 ; 0.82 ; 0.65)$ for $n=(0 ; 0.5 ; 1)$. Then, the rotational period of the star is defined through

$P=2 \pi \sqrt{I / 2 T}$. 
Thus, through (3), (5) and (8), the total self-energy and the rotational period can be expressed entirely in terms of $e, \bar{m}, R_{0}$ and $\kappa_{n}$.

As the star spins down due to magnetic braking, it follows a quasi-equilibrium sequence of constant baryonic mass (we do not consider significant mass-loss from the pulsar). Along this sequence, the total self-energy and rotational period, for a given equation of state, are then functions of the eccentricity only. In order to derive useful relations, we employ a series expansion of the structure of rotating compressible spheroids, around the nonrotating configuration, in powers of the eccentricity $e$, and retain terms of order at most six in $e$, a quite accurate, indeed, approximation for rotational periods of a few milliseconds. The total self-energy and angular velocity $\Omega=2 \pi / P$ are then written as

$$
\begin{aligned}
\mathcal{E}= & -\frac{3-n}{5-n} \frac{G \bar{m}^{2}}{R_{0}}\left[1-\frac{2}{5(3-n)} e^{2}-\frac{9}{35(3-n)} e^{4}\right. \\
& \left.-\frac{2\left(2050-1383 n+227 n^{2}\right) e^{6}}{2625(3-n)^{3}}+O_{8}\right], \\
\Omega= & e \sqrt{\frac{2 G \bar{m}}{|5-n| \kappa_{n} R_{0}^{3}}\left[1-\frac{3(11 n-5)}{70(3-n)} e^{2}\right.} \\
& -\frac{9\left(25+982 n-416 n^{2}\right)}{9800(3-n)^{2}} e^{4}
\end{aligned}
$$$$
-\frac{\left(3490875+12039717 n-11829047 n^{2}+\frac{7669405}{3} n^{3}\right)}{7546000(3-n)^{3}} e^{6}
$$

$\left.+O_{8}\right]$

Inverting the series expansion of $\Omega$, one obtains the following expansion for the eccentricity:

$$
\begin{aligned}
e=\frac{\tilde{\Omega}}{\sqrt{2}}[1 & +\frac{3(11 n-5)}{140(3-n)} \tilde{\Omega}^{2}+\frac{397 n^{2}+414 n+225}{2800(3-n)^{2}} \tilde{\Omega}^{4} \\
& \left.+O_{6}\right]
\end{aligned}
$$

where

$$
\tilde{\Omega}=\Omega \sqrt{\frac{|5-n| \kappa R_{0}^{3}}{G \bar{m}}},
$$

is a dimensionless form of the angular velocity. Taking time derivatives (denoted by an overdot), one can express the mass-energy loss $\dot{\mathcal{E}}$ in terms of the spin-down rate $\dot{P}$ :

$$
\begin{aligned}
\dot{\mathcal{E}}= & -4 \pi^{2} I_{0} \frac{\dot{P}}{P^{3}}\left[1+\frac{6 \pi^{2}(5+n)}{5(3-n)} \frac{1}{\tilde{P}^{2}}\right. \\
& \left.+\frac{4 \pi^{4}\left(275+204 n+n^{2}\right)}{35(3-n)^{2}} \frac{1}{\tilde{P}^{4}}+O_{6}\right],
\end{aligned}
$$

where $\tilde{P}=2 \pi / \tilde{\Omega}$ is a dimensionless form of the rotational period and $I_{0}=\frac{2}{5} \kappa_{n} \bar{m} R_{0}^{2}$ is the moment of inertia of a nonrotating star of baryonic mass $\bar{m}$ and radius
$R_{0}$. With this definition, $R_{0}$ remains constant during spindown (when changes in the baryonic mass are neglected), while the equatorial and polar radii can both change due to the changing eccentricity.

\section{Orbital period change}

The gravitational mass $m$ of a compact star (entering the gravitational equations of motion) can be decomposed (Spyrou 1977) as

$m=\bar{m}+\frac{\mathcal{E}}{c^{2}}$,

where $\bar{m}$ is the baryonic rest mass and $\mathcal{E}$ is the Newtonian self-energy, i.e

$\mathcal{E}=W+U+T$

We consider the 1PN motion of a binary system, consisting of two compact objects of gravitational masses $m_{\mathrm{p}}$ (primary) and $m_{\mathrm{c}}$ (companion). The orbital period of the binary system of total mass $M=m_{\mathrm{c}}+m_{\mathrm{p}}$ and orbital energy $E=\mu E_{0}$ (where $E_{0}$ is the orbital energy per unit reduced gravitational mass) is (Blanchet \& Schäfer 1989)

$P_{\mathrm{b}}=\frac{2 \pi G M \mu^{3 / 2}}{(-2 E)^{3 / 2}}\left[1+\frac{1}{4}\left(\frac{\mu}{M}-15\right) \frac{E}{\mu c^{2}}\right]$,

and $\mu=m_{\mathrm{c}} m_{\mathrm{p}} /\left(m_{\mathrm{c}}+m_{\mathrm{p}}\right)$ is the reduced gravitational mass of the system. If the primary loses energy isotropically and the orbital angular momentum is conserved, then it has been shown by Jeans $(1924,1928)$, assuming massenergy equivalence and Keplerian orbital motion, that the induced change in the binary's orbital period is

$\frac{\dot{P}_{\mathrm{b}}}{P_{\mathrm{b}}}=-2 \frac{\dot{m}_{\mathrm{p}}}{M}$.

It is easy to show that under our assumptions, the $1 \mathrm{PN}$ term on the right-hand side of (17) does not contribute to 1PN order in (18).

We notice that, the pulsar's spin can be included in the equations describing the orbital motion, following, e.g. the formalism of Gergely et al. (1998). In the Appendix, we show, that including the spin-orbit coupling, the total change in the binary's orbital period is

$\frac{\dot{P}_{\mathrm{b}}^{\mathrm{S}}}{P_{\mathrm{b}}}=-2 \frac{\dot{m}_{\mathrm{p}}}{M}-\frac{3 G(2+\eta) \cos \psi}{2 c^{2} a^{3 / 2} \sqrt{G M\left(1-\epsilon^{2}\right)}} \dot{S}$,

where $\eta=m_{\mathrm{c}} / m_{\mathrm{p}}, \psi$ is the angle substended by the orbital angular momentum $\boldsymbol{L}$ and the pulsar's spin $\boldsymbol{S}, a$ and $\epsilon$ are the semi-major axis and eccentricity of the orbit and $S$ is the magnitude of the spin. In (19), only the dominant, to 1PN order, spin-terms are included. In the Appendix, we also show that, for typical millisecond binary pulsars, the spin-term in the right-hand side of (19) is of order $\left(R_{0} / a\right)^{3 / 2}$ smaller than the first term. Since we will not be concerned with binaries that are near coalescence, in the remainder of this paper, we will neglect the spin-term in (19) and use only Eq. (18). 
Assuming that the spin-down is mainly due to magnetic braking and, thus, neglecting changes in the baryonic mass of the primary, we find

$\dot{m}_{\mathrm{p}}=\frac{\dot{\mathcal{E}}}{c^{2}}$,

and through (14), (15) and (18), we derive our final expression for the orbital period change due to spin-down

$$
\begin{aligned}
\frac{\dot{P}_{\mathrm{b}}^{\mathrm{S}}}{P_{\mathrm{b}}}= & \frac{8 \pi^{2} I_{0}}{c^{2} M} \frac{\dot{P}}{P^{3}}\left[1+\frac{6 \pi^{2}(5+n)}{5(3-n)} \frac{1}{\tilde{P}^{2}}\right. \\
& \left.+\frac{4 \pi^{4}\left(275+204 n+n^{2}\right)}{35(3-n)^{2}} \frac{1}{\tilde{P}^{4}}+O_{6}\right],
\end{aligned}
$$

This expression depends only on the spin and spin-down rate of the primary, the total mass $M$ of the binary system and the moment of inertia of a nonrotating star of same baryonic mass as the primary. To lowest order in the eccentricity, our result agrees with slow-rotation expressions derived previously in the literature (see Smarr \& Blandford 1976; Will 1981; Damour \& Taylor 1991) ${ }^{1}$, and extends those results to rapidly rotating stars. As we will show in the remainder of this paper, the higher order rotational corrections, that we introduce in (21), are essential, if one wants to arrive at quantitative constraints on the structure of compact objects, using observationally derived data of the above effect.

\section{Lowest order estimates}

Before applying (21) to specific binary systems, it is instructive to use the lowest-order term

$$
\left(\frac{\dot{P}_{\mathrm{b}}^{\mathrm{S}}}{P_{\mathrm{b}}}\right)_{0} \simeq \frac{8 \pi^{2} I_{0}}{c^{2} M} \frac{\dot{P}}{P^{3}},
$$

for obtaining a qualitative conclusion about the possible types of binary systems, in which the orbital change due to spin-down can be observationally important. For the effect to be measurable, it must be larger than achievable timing precision. For example, the precision with which the orbital decay $\dot{P}_{\text {orb }} / P_{\text {orb }}$ (due to quadrupole gravitational radiation emission) of the binary pulsar B1913+16 has been measured is $2.310^{-19} \mathrm{~s}^{-1}$ (Taylor 1992, 1993; Kaspi 1999).

Assuming a radius of $R_{0}=12 \mathrm{~km}$, a spin period of $1.6 \mathrm{~ms}$ and assuming that $m_{\mathrm{c}} \ll m_{\mathrm{p}}$, we see that the present effect exceeds the above precision, if $\dot{P}>$ $310^{-20} \mathrm{~s} / \mathrm{s}$. In the next section, we will show that including the higher order corrections in rotation, relaxes this requirement. Typical values of $\dot{P}$ for pulsars with periods of a few milliseconds are $10^{-21} \mathrm{~s} / \mathrm{s}<\dot{P}<10^{-19} \mathrm{~s} / \mathrm{s}$ (Phinney \& Kulkarni 1994; Lorimer 1998). Thus, we conclude that in binary systems, in which the primary is a rapidly rotating millisecond pulsar with period of a few

\footnotetext{
1 Note that, the first two references contain a missprint that was corrected in the third reference.
}

milliseconds only and spin-down rate larger than a few times $10^{-20} \mathrm{~s} / \mathrm{s}$, the orbital change due to the pulsar's spin-down becomes, in principle, observable. In contrast, the secular increase in the orbital period of the relativistic binary pulsar B1913+16 (due to the pulsar's spindown) is several orders of magnitude smaller than the secular decrease caused by emission of gravitational radiation, because of the pulsar's relatively large spin period of $59 \mathrm{~ms}$ (see Spyrou 1987 for a related discussion - an upper limit to self-energy-loss effects has still not been reached by current observational accuracy). Hence, in the case of B1913+16 and similar relativistic binary systems, the influence of self-energy losses on the orbital period are still beyond reach of experimental verification.

\section{Results for typical binary pulsars}

We now apply Eq. (21) to different possible millisecond pulsars in binary systems. We use the accuracy in measuring changes in the orbital period of PSR B1913+16, as a measure of the observability of the changes induced by spin-down. Thus, we define the ratio

$\Lambda=\frac{\dot{P}_{\mathrm{b}}^{\mathrm{S}} / P_{\mathrm{b}}}{2.310^{-19} \mathrm{~s}^{-1}}$

We note that, the measured value of $\dot{P} / P$ for millisecond pulsars is normally corrected by a galactic acceleration term. In the case of PSR B1913+16, the uncertainty in estimating the galactic acceleration term in $\dot{P} / P$ is $1.910^{-19} \mathrm{~s}^{-1}$ (Damour \& Taylor 1991), which is slithgly less than the presently achieved accuracy in measuring $\dot{P} / P$ for this pulsar.

In order to illustrate the importance of including the higher-order rotational terms, we define

$\Delta=\frac{\dot{P}_{\mathrm{b}}^{\mathrm{S}}-\left(\dot{P}_{\mathrm{b}}^{\mathrm{S}}\right)_{0}}{\dot{P}_{\mathrm{b}}^{\mathrm{S}}}$

as being the relative difference between the result (21) (which includes terms up to $\mathrm{O}_{4}$ ) and the lowest order estimate (22). We focus on typical millisecond pulsars of mass $m_{\mathrm{p}}=1.41 M_{\odot}$ using two different equations of state that span the expected range of possible realistic EOSs: a relatively stiff EOS, with polytropic index $n=0.5$ and a relatively soft EOS, with polytropic index $n=1.0$. For the stiff EOS, we choose the nonrotating radius to be $R_{0}=12 \mathrm{~km}$, while for the soft EOS, the nonrotating radius is chosen to be $R_{0}=10 \mathrm{~km}$. In this way, the corresponding nonrotating moment of inertia $I_{0}$ is, in both cases, in agreement with the relativistic moment of inertia computed by Ravenhall \& Pethick (1994) for various equations of state. Rapidly rotating neutron stars may have mass larger than 1.4 $M_{\odot}$, if they are spun-up by accretion. However, the neutron star's mass enters in the r.h.s. of Eq. (21) only through the ratio $I_{0} / M \sim R_{0}^{2}$, which is much more sensitive to the radius of the star than to its mass. Thus, it is sufficient, in the present context, to consider only one representative value for the mass. 
Table 1. Relative rate of orbital period change (second column), corresponding to different values of the pulsar's period derivative $\dot{P}$. Two different cases for the pulsar's period are considered. The quantity $\Delta$ measures the significance of the higher-order rotational corrections, while $\Lambda$ is the ratio of the second column to the accuracy achieved in measuring orbital period changes in the binary pulsar B1913+16. The pulsar's mass is $m_{\mathrm{p}}=1.41 M_{\odot}$, while the mass of the companion is $m_{\mathrm{c}}=0.2 M_{\odot}$. Results are for a stiff equation of state, of polytropic index $n=0.5$

Stiff EOS $(n=0.5)$

\begin{tabular}{|c|c|c|c|}
\hline $\begin{array}{c}\dot{P} \\
\mathrm{~s} / \mathrm{s}\end{array}$ & $\begin{array}{c}\dot{P}_{\mathrm{orb}}^{\mathrm{S}} / P_{\text {orb }} \\
\left(\mathrm{s}^{-1}\right)\end{array}$ & $\begin{array}{c}\Delta \\
(\%)\end{array}$ & $\Lambda$ \\
\hline \multicolumn{4}{|c|}{$P=1.6 \mathrm{~ms}$} \\
\hline $110^{-18}$ & $1.310^{-17}$ & 32 & 56.4 \\
\hline $510^{-19}$ & $6.510^{-18}$ & 32 & 28.2 \\
\hline $110^{-19}$ & $1.310^{-18}$ & 32 & 5.6 \\
\hline $510^{-20}$ & $6.510^{-19}$ & 32 & 2.8 \\
\hline $110^{-20}$ & $1.310^{-19}$ & 32 & 0.6 \\
\hline \multicolumn{4}{|c|}{$P=3 \mathrm{~ms}$} \\
\hline $510^{-18}$ & $7.410^{-18}$ & 10 & 32.4 \\
\hline $110^{-18}$ & $1.510^{-18}$ & 10 & 6.5 \\
\hline $510^{-19}$ & $7.410^{-19}$ & 10 & 3.2 \\
\hline $110^{-19}$ & $1.510^{-19}$ & 10 & 0.7 \\
\hline $510^{-20}$ & $7.410^{-20}$ & 10 & 0.3 \\
\hline
\end{tabular}

The mass of the companion is fixed at $m_{\mathrm{c}}=0.2 M_{\odot}$, which is a typical value for white-dwarf companions of millisecond pulsars in binary systems (our results are weakly dependent on the mass of the companion, if the companion is a low-mass white dwarf). We do not consider the case of binary systems with two neutron stars, as, in that case, changes in the orbital period are dominated by quadrupole gravitational radiation losses (see Spyrou \& Kokkotas 1994, for an account of self-energy loss effects on the gravitational radiation from neutron star binaries).

Table 1 displays our results for the stiff EOS. For $P=1.6 \mathrm{~ms}$ (eccentricity $e=0.517$ ), $\Lambda=56.4$ for $\dot{P}=10^{-18} \mathrm{~s} / \mathrm{s}$, while the value $\Lambda=5.6$ for $\dot{P}=10^{-19} \mathrm{~s} / \mathrm{s}$ is also interesting for observations. Notice the large relative difference of $\Delta=32 \%$, which demonstrates the importance of the higher-order rotational effects. For $P=3 \mathrm{~ms}$ (eccentricity $e=0.273$ ),$\Lambda=32.4$ for $\dot{P}=510^{-18} \mathrm{~s} / \mathrm{s}$ and $\Lambda=3.2$ for $\dot{P}=510^{-19} \mathrm{~s} / \mathrm{s}$, while, for $\dot{P}<10^{-19} \mathrm{~s} / \mathrm{s}$, the magnitude of the effect is less than the presently achieved observational accuracy. For the larger rotational period, the effect of higher-order rotational terms is reduced to $\Delta=10 \%$.
Table 2. Same as Table 1, but for a soft equation of state of polytropic index $n=1.0$

Soft $\operatorname{EOS}(n=1.0)$

\begin{tabular}{|c|c|c|c|}
\hline $\begin{array}{c}\dot{P} \\
\mathrm{~s} / \mathrm{s}\end{array}$ & $\begin{array}{c}\dot{P}_{\mathrm{orb}}^{\mathrm{S}} / P_{\mathrm{orb}} \\
\left(\mathrm{s}^{-1}\right)\end{array}$ & $\begin{array}{c}\Delta \\
(\%)\end{array}$ & $\Lambda$ \\
\hline \multicolumn{4}{|c|}{$P=1.6 \mathrm{~ms}$} \\
\hline $110^{-18}$ & $6.010^{-18}$ & 19 & 26.1 \\
\hline $510^{-19}$ & $3.010^{-18}$ & 19 & 13.1 \\
\hline $110^{-19}$ & $6.010^{-19}$ & 19 & 2.6 \\
\hline $510^{-20}$ & $3.010^{-19}$ & 19 & 1.3 \\
\hline $110^{-20}$ & $6.010^{-20}$ & 19 & 0.3 \\
\hline \multicolumn{4}{|c|}{$P=3 \mathrm{~ms}$} \\
\hline $510^{-18}$ & $3.910^{-18}$ & 6 & 17.0 \\
\hline $110^{-18}$ & $7.810^{-19}$ & 6 & 3.4 \\
\hline $510^{-19}$ & $3.910^{-19}$ & 6 & 1.7 \\
\hline $110^{-19}$ & $7.810^{-20}$ & 6 & 0.3 \\
\hline $510^{-20}$ & $3.910^{-20}$ & 6 & 0.2 \\
\hline
\end{tabular}

Table 2, displays the corresponding results for the soft EOS. For $P=1.6 \mathrm{~ms}$ (eccentricity $e=0.332$ ), $\Lambda=26.1$ for $\dot{P}=10^{-18} \mathrm{~s} / \mathrm{s}$, while $\Lambda=2.6$ for $\dot{P}=10^{-19} \mathrm{~s} / \mathrm{s}$, roughly a factor of two smaller than for the stiff EOS. The rotational effects are reduced to $\Delta=19 \%$, For $P=3 \mathrm{~ms}$ (eccentricity $e=0.175$ ),$\Lambda=17.0$ for $\dot{P}=510^{-18} \mathrm{~s} / \mathrm{s}$ and $\Lambda=1.7$ for $\dot{P}=510^{-19} \mathrm{~s} / \mathrm{s}$. The rotational effects are now only $\Delta=6 \%$.

It is evident that the results are sensitive to the chosen equation of state. The present uncertainty in the highdensity equation of state of neutron star matter (which could be as stiff as a $n=0.5$ polytrope or as soft as a $n=1.0$ polytrope) introduces, in the computed orbital period change, an unknown factor, which could be as large as a factor of two. A measurement of the orbital change due to the pulsar's spin-down and a knowledge (or guess) of it's baryonic mass could yield a value for (or constrain) the moment of inertia $I_{0}$ of nonrotating neutron stars of same mass $m_{\mathrm{p}}$. Combined with a measurement (or educated guess) of the pulsar's mass, this information can then constrain the very high-density equation of state of compact stars.

Of interest, currently, is the search for sub-millisecond pulsars, because the observation of such an object would drastically restrict the choice of the possible equation of state for compact objects ${ }^{2}$. We do not consider in detail millisecond pulsars rotating faster than $1.6 \mathrm{~ms}$ (roughly

\footnotetext{
${ }^{2}$ We thank the referee, Dr. U. Geppert for kindly pointing out to us the possible importance of sub-millisecond pulsars in the context of the present paper.
} 
the period of the fastest observed millisecond pulsar, todate) for two reasons. Firstly, because millisecond pulsars created by accretion-induced spin-up can be unstable to the emission of gravitational waves, through the growth of $r$-mode oscillations (Andersson et al. 2000). This instability limits the minimum expected period of millisecond pulsars to roughly $1.5 \mathrm{~ms}$, if neutron stars are not composed of strange matter. Secondly, because new odservational attempts of finding millisecond pulsars are now significantly more sensitive than previous observations (which used to be limited to periods larger than about $1.5 \mathrm{~ms}$ ). In spite of this improved sensitivity, no faster millisecond pulsars have been found todate (Crawford et al. 2000). Nevertheless, should such faster millisecond pulsars be observed in binary systems in the future (which is not excluded, if, for example, pulsars are composed of strange matter, see e.g. Stergioulas et al. 2000), then our main result, Eq. (21), implies that both the importance of higherorder rotational corrections and the orbital period change would be even more significant than for our presently considered rotational periods. For reasons of completeness and for future probable purposes, we note that, in the case of a pulsar with e.g. a $0.8 \mathrm{~ms}$ period and the same values of $\dot{P}$ as in Table 1, the orbital-period change, due to spin-down, would be roughly 8 times larger than for a $1.6 \mathrm{~ms}$ pulsar.

\section{Discussion}

We have extended previous, approximate, calculations of the orbital period change in binary pulsars (due to the pulsar's spin-down), by including higher-order rotational effects and the influence of the choice of the high-density equation of state. We have shown that the above higherorder terms, as well as the choice of the equation of state are significant, if quantitative constraints are to be drawn for future observations of this effect. We also showed, that the inclusion of the pulsar's spin in the description of the binary's orbit, does not contribute significantly to the orbital period change. Our treatment relies on the description of rotating stars as compressible perfect fluid spheroids (ignoring the possible influence of a solid crust), in which one can express both the change in spin-period and the change in radius, during spin-down, through the change in the eccentricity of the spheroid. Binary pulsars, that are interesting for the above effect to be measurable, must be rapidly rotating, with a rotational period of only a few $\mathrm{ms}$ and, in addition, with a period derivative equal to or larger than a few times $10^{-20} \mathrm{~s} / \mathrm{s}$. As Phinney \& Kulkarni (1994) point out, a millisecond pulsar like B1937+21 (which has $P=1.56 \mathrm{~ms}$ and $\dot{P}=10^{-19} \mathrm{~s} / \mathrm{s}$ ), if it were in a binary system, would yield a measurable orbital period change. Using our results, i.e. Eq. (21), one could then derive quantitative constraints on the pulsar's moment of inertia. More accurate constraints could be derived, using numerical models of rapidly rotating neutron stars that satisfy the general-relativistic field equations, for various realistic equations of state. Such a computa- tion, however, will make sense only after observational measurements of the predicted effect will start becoming available. The increasing number of millisecond pulsars discovered in binary systems (Camilo et al. 2000) is a positive sign that this could happen in the near future.

Acknowledgements. We thank Bernard F. Schutz, Gerhard Schäfer, Kostas Kokkotas and Theocharis Apostolatos for helpful comments and suggestions and John-H. Seiradakis for a careful reading of the manuscript. This research was supported by the European Union grant HPRN-CT-2000-00137.

\section{Appendix}

We have shown that (18) depends only on the Newtonian orbital characteristics (as the $1 \mathrm{PN}$ orbital terms contribute at $2 \mathrm{PN}$ order). Thus, the pulsar's spin can be included in the description of the binary's orbit, following the formalism of Gergely et al. (1998), who consider the correction to the Newtonian orbit, induced by the spin. We define $\eta=m_{\mathrm{c}} / m_{\mathrm{p}}, \psi$ the angle substended by the orbital angular momentum $\boldsymbol{L}$ and the pulsar's spin $\boldsymbol{S}, a$ and $\epsilon$ are respectively the semi-major axis and eccentricity of the pulsar's orbit and $L, S$ the magnitudes of its orbital angular momentum and spin, respectively. Then, the binary's period $P_{\mathrm{b}}$, orbital energy $E$ and semi-major axis $a$, are given by

$$
\begin{aligned}
P_{\mathrm{b}} & =2 \pi \frac{G M \mu^{3}}{(-2 \mu E)^{3 / 2}} \\
E & =-\frac{G M \mu}{2 a}\left[1+\frac{G S(2+\eta) \cos \psi}{c^{2} a^{3 / 2} \sqrt{G M\left(1-\epsilon^{2}\right)}}\right],
\end{aligned}
$$

and

$a=-\frac{G M \mu}{2 E}\left[1-\frac{2 E S(2+\eta)}{c^{2} M L} \cos \psi\right]$

In the case that the spin is zero and the star loses massenergy isotropically (e.g. due to thermal emission), it has been shown by Jeans $(1924,1928)$ that the product $A=$ $a M$ remains constant during the orbit's evolution (which leads to Eq. (18) in the text). We treat the inclusion of spin in the description of the orbital motion as a first-order perturbation about the orbital motion with zero spin. If so, we can write

$E=\mathcal{E}_{0}(1+\delta \tilde{E})$

$A=A_{0}(1+\delta \tilde{A})$,

where

$\delta \tilde{E}=\frac{G S(2+\eta) \cos \psi}{c^{2} a^{3 / 2} \sqrt{G M\left(1-\epsilon^{2}\right)}}$,

$\delta \tilde{A}=-\frac{2 E S(2+\eta)}{c^{2} M L} \cos \psi-\delta \tilde{E}$

and $\mathcal{E}_{0}, A_{0}$ are the the values of $E$ and $A$ when spin-orbit coupling is ignored. Then,

$\frac{\dot{P}_{\mathrm{b}}^{\mathrm{S}}}{P_{\mathrm{b}}}=-2 \frac{\dot{m}_{\mathrm{p}}}{M}+\frac{3}{2}(\dot{\delta} \tilde{A}-\dot{\delta} \tilde{E})$. 
Keeping only first-order terms in the spin (and keeping only $1 \mathrm{PN}$ terms), we find

$$
\frac{\dot{P}_{\mathrm{b}}^{\mathrm{S}}}{P_{\mathrm{b}}}=-2 \frac{\dot{m}_{\mathrm{p}}}{M}-\frac{3 G(2+\eta) \cos \psi}{2 c^{2} a^{3 / 2} \sqrt{G M\left(1-\epsilon^{2}\right)}} \dot{S}
$$

In order to arrive at an order-of-magnitude estimate for the spin-term in (33), we substitute the lowest-order (in spin-period) expressions derived in the main text (using $S=I \Omega$ ), and find

$$
\begin{aligned}
\frac{\dot{P}_{\mathrm{b}}^{\mathrm{S}}}{P_{\mathrm{b}}} & =\frac{8 \pi^{2} I_{0}}{c^{2} M} \frac{\dot{P}}{P^{3}} \\
& \times\left[1+\frac{3}{8} \frac{(2+\eta) \cos \psi}{e \sqrt{1-\epsilon^{2}}} \sqrt{2|5-n| \kappa_{n}}\left(\frac{R_{0}}{a}\right)^{3 / 2}\right] .
\end{aligned}
$$

Since the spin-term is of order $\left(R_{0} / a\right)^{3 / 2}$ smaller than the isotropic term in (34), it can be neglected for typical binary pulsar systems considered in the present paper.

\section{References}

Andersson, N., Jones, D. I., Kokkotas, K. D., \& Stergioulas, N. 2000, ApJL, 534L, 75

Blanchet, L., \& Schäfer, G. 1989, MNRAS, 239, 845

Camilo, F., Lorimer, D. R., Freire, P., Lyne, A. G., \& Manchester, R. N. 2000, ApJ, 535, 975

Caporali, A., \& Spyrou, N. 1981, Gen. Rel. Grav., 13, 689

Chandrasekhar, S. 1969, Ellipsoidal Figures of Equilibrium (Yale University Press, New Haven, Connecticut)

Crawford, F., Kaspi, V., \& Bell, J. F. 2000, ApJ, 119, 2376

Damour, T., \& Taylor, J. H. 1991, ApJ, 366, 501

Epstein, R., \& Wagoner, R. V. 1975, ApJ, 197, 717

Gergely, L. A., Perjés, Z. I., \& Vasúth, M. 1998, Phys. Rev. D., 57,3423
Jeans, J. H. 1924, MNRAS, 84, 2

Jeans, J. H. 1928, Astronomy and Cosmogony (Cambridge U. Press)

Kaspi, V. M. 1999, General relativity and relativistic astrophysics: Eighth Canadian conference, Montreal, Quebec, June 1999, ed. C. P. Burgess, \& R. C. Myers (Melville, N.Y.: AIP Conf. Proc. 493), 3 [astro-ph/9912285]

Lai, D., Rasio, F. A., \& Shapiro, S. L. 1993, ApJSu, 88, 205

Lorimer, D. R. 1998, Living Reviews in Relativity, vol. 1, 199810, http://www. livingreviews.org

Peters, P. C., \& Mathews, J. 1963, Phys. Rev., 131, 435

Phinney, E. S., \& Kulkarni, S. R. 1994, ARA\&A, 32, 591

Ravenhall, D. G., \& Pethick, C. J. 1994, ApJ, 424, 846

Smarr, L. L., \& Blandford, R. 1976, ApJ, 207, 574

Spyrou, N. 1977a, Gen. Rel. Grav., 7, 463

Spyrou, N. 1977b, Gen. Rel. Grav., 7, 491

Spyrou, N. 1978, Gen. Rel. Grav., 9, 519

Spyrou, N. 1981, Gen. Rel. Grav., 13, 473

Spyrou, N. 1983, Gen. Rel. Grav., 15, 363

Spyrou, N. 1985, A\&A, 149, 283

Spyrou, N. 1987, A\&A, 174, 355

Spyrou, N. 1988, J. Astrophys. Asr., 9, 25

Spyrou, N. 1999, Accretion Processes in Astrophysical Environments, ed. P. G. Niarchos (University of Athens), 49

Spyrou, N., \& Papadopoulos, D. 1985, Gen. Rel. Grav., 117, 1059

Spyrou, N., \& Kokkotas, K. D. 1994, ApJ, 431, 254

Stergioulas, N., Kluzniak, W., \& Bulik, T. 1999, A\&A, 352L, 116

Taylor, J. H. 1992, Philos. Trans. Roy. Soc. London A, 341, 117

Taylor, J. H. 1993, Particle Astrophysics, IVth Rentrées de Blois, ed. G. Fontaine, \& J. Tran Thanh Van (Gif-surYvette, France: Éditions Frontières), 367

Weisberg, J. M., \& Taylor, J. H. 1984, Phys. Rev. Lett., 52, 1348

Will, C. M. 1981, Theory and Experiment in Gravitational Physics (Cambridge University Press, Cambridge) 